\title{
LINKING THE SPATIAL SYNTAX OF COGNITIVE MAPS TO THE SPATIAL SYNTAX OF THE ENVIRONMENT
}

YOUNG OOK KIM $i$ is an associate professor of architectural and urban morphology at the University of Sejong, Seoul. He is a member of the Korean Institute of Architects and is the director of Space Syntax Korea.

ALAN PENN is a professor of architectural and urban computing at The Bartlett, University College of London, where he is the director of the Virtual Reality Centre for the Built Environment. His research spans the fields of architecture, urban design, and computing, with a focus on developing an understanding of interactions between individuals, social and organizational groups, and the built environments they inhabit.

ABSTRACT: This article investigates the effects of the spatial configuration of the local environment on residents' spatial cognitions of their built environment by examining the relationship between the spatial syntax of sketch maps and the spatial syntax of the environment. Hampstead Garden Suburb in London was investigated in detail. Structured interview surveys were carried out to elicit residents' sketch maps of their local area. Analysis of the spatial characteristics of the area and of the sketch maps using space-syntax methods provided a common basis for analyses of these data. Strong correlations were identified between residents' sketch maps and the spatial configuration of the area. The degree of local integration of spatial configuration is the most significant factor in relations with the two variables of sketch maps, the frequency of appearance of configurational elements, and the global syntactic characteristics of spatial configuration in sketch maps. Findings suggest that spatial syntax of configuration in real environments and spatial syntax of cognitive maps in spatial cognition are closely related.

Keywords: space syntax; sketch map; cognitive map; spatial cognition; way finding

The literature on human cognition suggests that configurational aspects of the environment have significant cognitive consequences. Lynch (1960) notes that to be "imageable," an area needs to be apprehended as a pattern of 
high continuity with a number of distinctive but interconnected parts. Hillier (1996) has argued that spatial configuration may place constraints on spatial experience because it encourages or impedes aspects of human activity through spatial cognition and subsequent behavior. Golledge and Stimson (1997) have also emphasized that the path or network structure used in everyday spatial behavior becomes a critical feature of the image of a spatial environment. Others suggest that spatial layout of the built environment influences the accuracy of cognitive representations of real-world spatial information (e.g., Appleyard, 1970; O’Neill, 1991). However, relatively few studies have incorporated configurational aspects of the environment and of their cognitive dimensions within a single framework in investigating the relationship between people and the built environment. In our view, there is thus a gap in our understanding of the relationship between configuration and cognitive representation.

There seem to be two causes for this gap. First, there has not been an analytical method for describing configurational characteristics of cognitive representations. Those studies that have investigated configurational characteristics objectively have mostly focused on the local characteristics of spatial configuration and not on their global relational context (e.g., Sadalla \& Montello, 1989). Methods for analyzing global characteristics have been descriptive rather than analytic (e.g., Weisman, 1981). This is perhaps not surprising because methods for analysis of relational properties of configurations - those relating parts to the whole pattern - are not widespread. In fact, use of the word global to describe those aspects of spatial configuration relating a part to the whole is not common currency in the cognitive field, although it is commonly used in this sense in syntactic approaches to spatial analysis. Second, mainly because of the different research viewpoints between syntactic approaches to spatial configuration and cognitive approaches to spatial configuration (e.g., O'Neill, 1991) the interaction between syntactic characteristics of spatial configuration and cognitive maps in spatial cognition has not been extensively explored.

The gap may be described in two ways. Firstly, it occurs through the neglect of perception-cognition studies within research based on syntactic descriptions of spatial configuration, ${ }^{1}$ and secondly, in a similar vein, it occurs through the neglect of analytic descriptions of spatial configuration in research into cognitive maps. In this context, this article investigates the relationship between spatial configuration and spatial cognition by adapting syntactic analysis of spatial configuration to represent and to quantify residents' sketch maps of their local area. This research takes an explicit theoretical position, using empirical data to investigate underlying regularities between 
the spatial and the cognitive dimensions. The main objectives of the research are twofold: (a) to investigate whether syntactic analysis methodology can be usefully adapted to the investigation of sketch map data and (b) to investigate the association between configurational features and cognitive representations and thus throw light on how configurational aspects of the built environment may affect cognitive representation. The article is structured in three parts: the first focuses on the syntactic analysis of configurational characteristics in the study area; the second presents the analysis of the residents' sketch maps; and the third investigates the relationship between these two.

\section{COGNITIVE REPRESENTATION OF SPATIAL CONFIGURATION IN SKETCH MAPS}

Hart and Moore (1973) define spatial cognition as "the knowledge and internal or cognitive representation of the structure, entities, and relations of space; in other words, the internalized reflection and reconstruction of space in thought" (p. 248). Similarly, Downs and Stea (1973) note that "the process of cognitive mapping is a means of structuring, interpreting, and coping with complex sets of information that exist in different environments" (pp. 9-10). The end product of the cognitive-mapping process is a cognitive map. The literature is less clear on the precise nature of the cognitive map itself. Two primary forms of representation are posited. The first comprises an explicit representation of routes; the second proposes that a more or less realistic map must be constructed because it is only this that can account for our ability to infer new routes and to relocate ourselves by reference to cues once lost. O' Keefe and Nadel (1978) provide anatomic evidence for the latter through their investigations of the function of the hippocampus. Whatever the internal form of representation, it is clear that people can provide more or less correct sketch maps of environments with which they are familiar from memory.

The process of sketch mapping and the map itself have been used as research tools to measure how people perceive and recognize their built environments and how people act in them. It has long appeared to be a useful instrument for recovering information about the way we represent the environment to ourselves. Although sketch maps are generally incomplete, distorted, and employ mixed metric or nonmetric modes of representation, they provide data, such as the number of features; the mix of point, line, and area features; and the topological relations of elements, including the sequences of cues along routes or the sequence of segments and turns along routes. Additional information can be obtained from the system constructed as 
the basis for sketch maps, particularly the regularity or irregularity of frameworks such as street systems, by using analytical tools to quantify their characteristics.

Sketch maps have mainly been analyzed in three ways hitherto. First, much research using sketch maps has involved investigation of the perception of local configurational elements in different environments and of the comparison of the perceived pattern, as recovered from the sketch map, to the real pattern. Sadalla and Magel (1980) investigated the effect of the number of turns in a path on the perception of its length. They found that the greater the number of turns in a path the greater its perceived length. Similarly, greater numbers of path intersections along a route have also been found to increase its estimated length (Sadalla \& Staplin, 1980). The prominence of a grid configuration in cognitive schemata of the physical environment is also suggested by several studies showing systematic distortions of nongrid structures. Evans and Pezdek (1980) found that common distortions include the straightening of gradual curves, the squaring of nonperpendicular intersections, and the aligning of nonparallel streets. At the building scale, there has been little empirical research concerned with the direct relationship between spatial configuration and its cognitive representations. Weisman's (1981) study suggests a tentative understanding of this link. Weisman rated the legibility of simplified floor plans, which are classified from abstracted twodimensional diagrams of real buildings into so-called high and so-called low groupings, and found these values to be good predictors of the building users' self-reported incidences of being lost. Users become disoriented in buildings where the overall circulation pattern is confusing and hard to imagine. Second, in reviewing studies of sketch maps, Pipkin (1981) notes that the contents of images are often treated as dependent variables in relation to factors such as socioeconomic status, length of residence, mobility characteristics, and the activity patterns of people (e.g., Appleyard, 1970). Third, frequency counts of the appearance of different features in sketch maps have been used to develop a composite map on which those places represented by the largest number of people are located (e.g., Lynch, 1960). Composite maps of this sort are proposed to provide a representation of the main significant features in an area for the given population. Most previous analyses of sketch maps have thus concentrated on the disaggregation and the counting of represented elements. Very few studies have focused on the global or the relational aspects of spatial configuration in sketch maps objectively, although many researchers refer to the role of spatial configuration in cognitive representation (e.g., Zimring, 1981).

Cognitive studies therefore provide us with useful methods but little in the way of a theoretical starting point for an inquiry into the roles of global (part- 
whole) aspects of spatial configuration in cognitive maps. This appears to be primarily because of the absence of methods to describe both spatial configuration of environments themselves and the more subjective configurations represented in sketch maps. Hart and Moore (1973), regarding the difficulty of describing configuration, argue that even though psychologists and geographers alike converge in treating the understanding of spatial configuration as the ultimate stage of spatial cognition, configuration is perhaps the most difficult aspect of the environment to describe in an objective and analytic manner. Methods that have been used to date in analyzing sketch maps (and so the cognitive maps that may underlie them) have thus neglected to describe spatial configuration as a whole entity. And yet it is exactly the relationship between the part of an environment that one can see at some instant, and the remainder that one cannot perceive directly, that must underlie any useful form of cognitive maps. The absence of a viable method for studying these part-whole spatial relationships appears, therefore, to constrain exploration of cognitive maps. If methods were available to represent and to quantify the part-whole relationships in spatial configurations, then we might be better able to understand human cognition of the built environment.

\section{THE BASIS OF CONFIGURATIONAL ANALYSIS}

The need to develop methods for representing part-whole relationships in spatial configurations has also figured in studies of the social function of the built environment. To describe and analyze these characteristics of spatial configuration, Hillier and Hanson (1984) developed a methodology for representing and for measuring the pattern properties of open space in the built environment. The axial map of an area is drawn on the basis of open-space structure in a plan. It consists of the fewest and the longest sets of lines of sight and of access that pass through all the open spaces in an urban area and minimizes the number of changes of direction between any other pair of lines. Figure 1 shows this procedure: the open space structure (a) and an axial $\operatorname{map}(\mathrm{b}) .^{2}$

Once an axial map is obtained, it can be used as the basis for calculation of various relational properties of the geometry of an environment. The second stage of the process involves transcribing the axial map as a graph where nodes represent lines and where edges depict their relations of intersection. The properties of this graph can then be measured in various ways. Because the elements of the graph (the nodes) represent longest lines of sight passing through parts of the local environment, and because the links in the graph represent transitions from one line to another and, thus, changes of direction, 
A

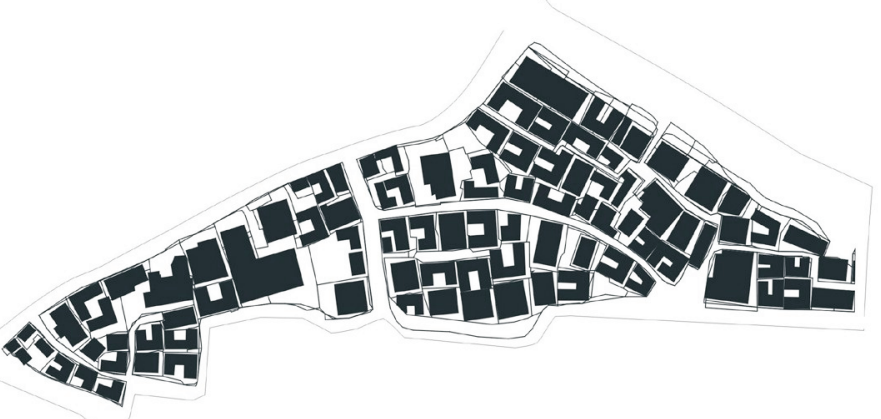

B

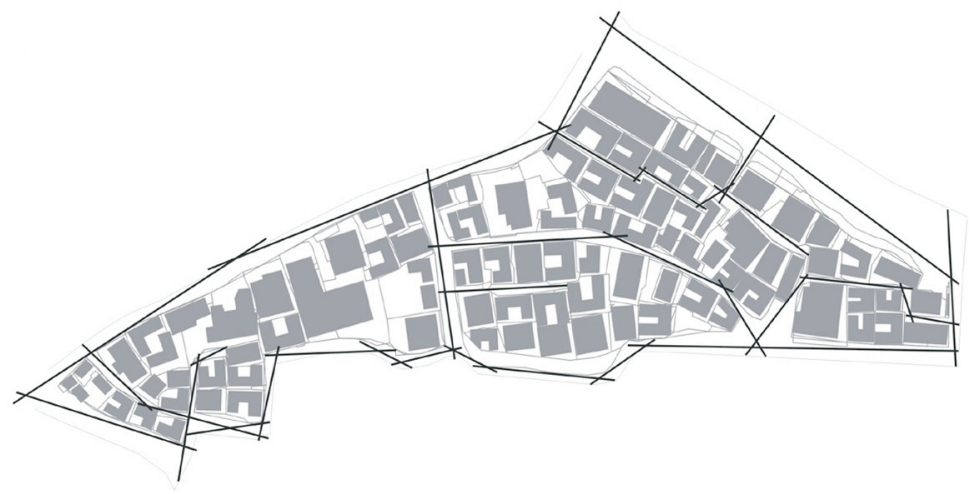

Figure 1: The Spatial Structure of Open Space (A) and Its Axial Representation (B)

measures of the graph have a direct relationship to certain aspects of the nature of the environment we experience as we move around.

The most important aspect of syntactic measurement for our present purposes relates to the distinction between local and global measures of the graph. A node in a graph is directly connected to some number of other nodes. This measure of connectivity corresponds in an axial map to the number of axial lines that intersect a particular line and is a purely local measure. 
That is, a line's connectivity can, in principle, be seen without moving off the line in question. Local measures are contrasted to global measures, such as the mean depth of a whole graph, from a particular axial line. The measure of mean depth, in principle, requires one to traverse the whole map, counting changes of direction, and building up knowledge of how many axial lines are how many changes of direction away. In this sense, they measure the global properties of the configuration. One can measure properties of the graph, such as mean depth, within any number of steps or radii of each line, in turn. The greater the radius, the more global the extent of the measure. Hillier (1996) describes how these measures can be relativized for the number of lines within that radius so that measures can be directly compared and calls these measures integration. ${ }^{3}$ Integration, measured to the full radius of the system under consideration, is called global integration. A more integrated line is one that is, on average, shallow to a larger number of lines, whereas a less integrated (or more segregated) line is deeper, on average. The local measure of integration used is radius 3 integration, which is called local integration.

\section{METHOD}

\section{SYNTACTIC DESCRIPTION OF HAMPSTEAD GARDEN SUBURB}

The study area in Hampstead Garden Suburb is located about $5 \mathrm{~km}$ north of central London. It is bounded by Finchley Road to the west and the A1, which consists of Falloden Way and Market Place, to the north. Hampstead Heath Extension defines the boundary of the suburb to the south, and The Bishops Avenue the boundary to the east (see Figure 3). A syntactic analysis of spatial configuration begins by representing the layout of the case study area as an axial map. Based on the analysis of the axial map described in the previous section, we then produce a global integration map of Hampstead Garden Suburb using integration values ranging from black for the most integrated line to light gray for the least integrated line. In the same way, we can produce a map of local (radius 3 ) integration. These integration maps allow the systematic quantification of certain aspects of the spatial structure of the area.

Figure 2 represents the global integration map of Hampstead Garden Suburb. The suburb is composed of 158 lines. The lines are represented from black to light gray in terms of their degrees of integration. The black lines are most integrated or have the fewest changes of direction from all others on 


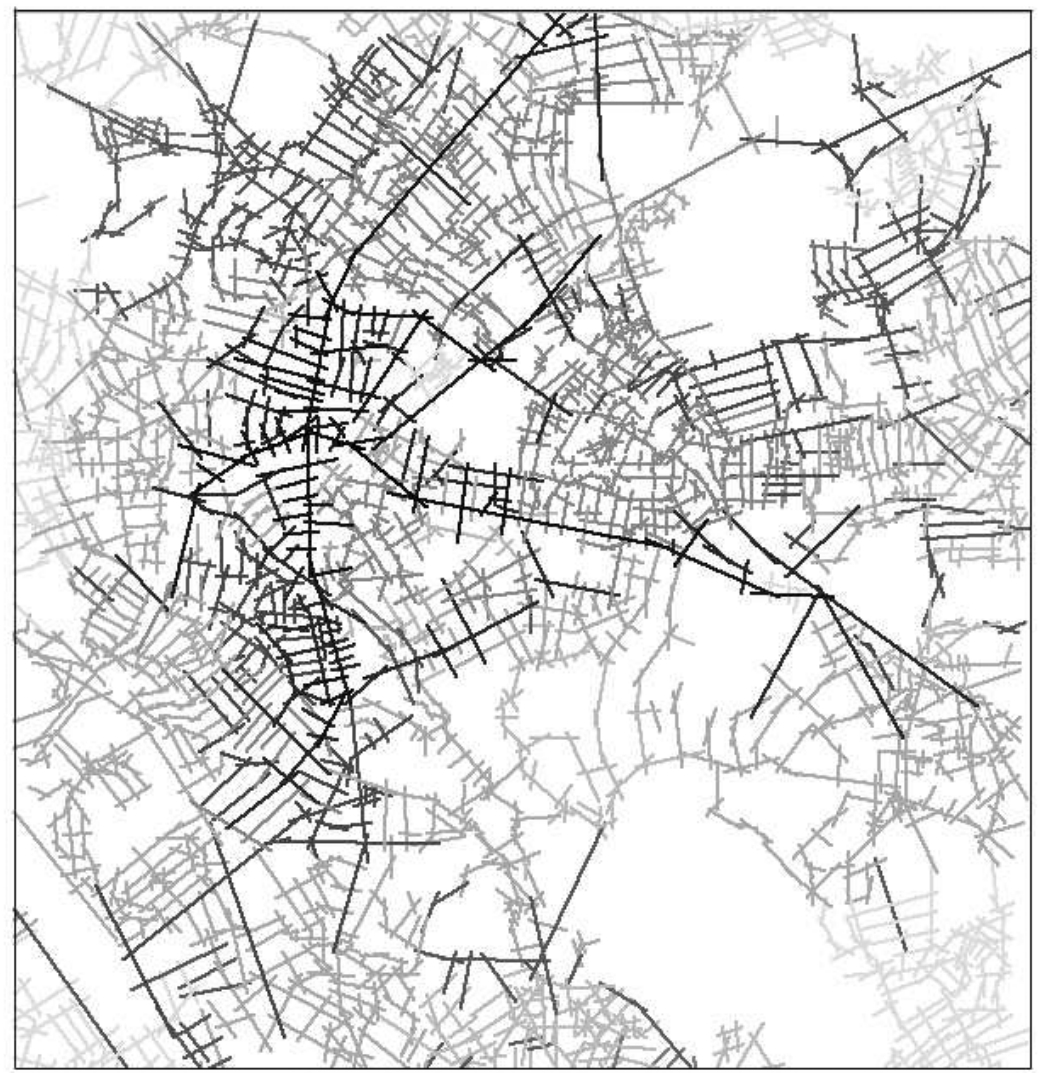

Figure 2: Global Integration Map of Hampstead Garden Suburb

average; the light gray are the most segregated. Hampstead Garden Suburb is located between two highly integrated spaces, the A1 and Finchley Road. Figure 3 illustrates the local integration map of the suburb; the map measures the average depth of spaces within the immediate neighborhood (syntactically three steps of depth away), and it shows how integrated or segregated each space is.

\section{PROCEDURE OF SKETCH MAPPING TASK}

An interview survey was conducted to obtain sketch maps of spatial layouts of the study area. It was based on a household sample of the residents in 


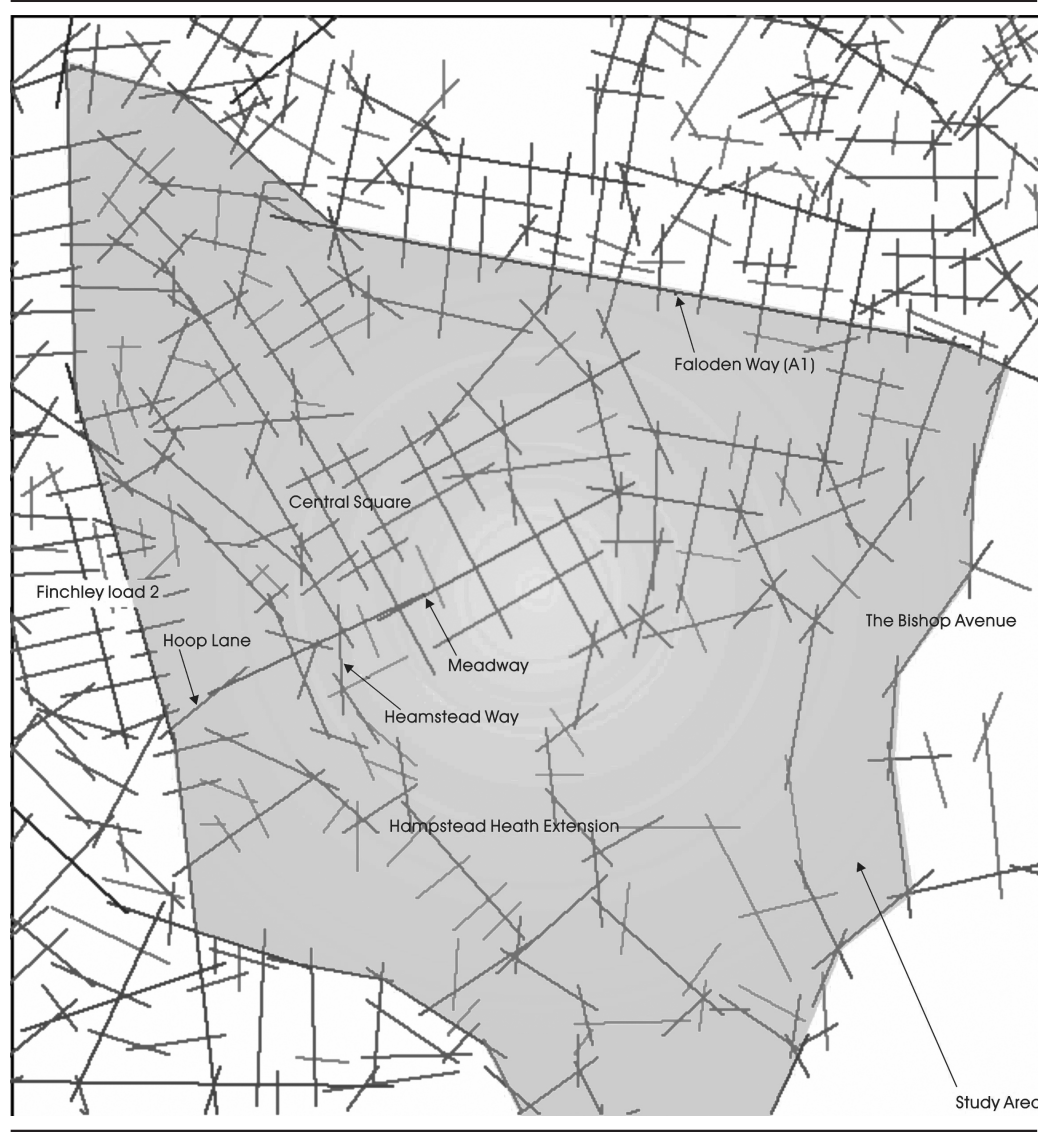

Figure 3: Local Integration Map of Hampstead Garden Suburb

Hampstead Garden Suburb. Letters were sent to randomly selected addresses asking for cooperation in the survey. One address was chosen randomly from all households identified on an axial line. Then, an interviewer visited and found an adult member of the household, and an appointment was made if he or she were willing to participate. Where participation was declined, a randomly selected replacement address was chosen on that line. The sample size was 76.

Respondents were asked to draw a sketch map of the spatial layout of Hampstead Garden Suburb for 20 minutes, including streets, buildings, and open spaces. The interviewee was instructed that the purpose of the sketch was as a guide for a visitor to orient himself or herself and to find his or her 
way in the suburb. To set a uniform scale and orientation to the map, two local stations were marked. One is situated on the lower left corner of the paper provided and the other on the right upper corner. The scale, given by distance between these two places, is 1:10,000. Participants were reminded to draw not only their neighborhood but the whole suburb. A wide range of competence in drawing maps was found among the participants. Figures $4 \mathrm{a}$ and $4 \mathrm{~b}$ shows a relatively well drawn and a poorly drawn sketch map side by side.

There were a number of typical errors, including incompleteness, variations in scale across the area, roads being drawn too wide, possible straightening of roads, and possible orthogonalization of roads. The maps are also sometimes too simple, highly selective, distorted, and augmented. These typical errors are valuable in this study because they may allow us to understand the syntactic characteristics of sketch maps that reflect how people perceive and represent the real environment. Errors that have been reported in other studies, such as drawing of discontinuous systems or use of single lines to represent streets, occurred only in few samples of participants because people were reminded not to do so in the beginning of an interview. The latter depiction has been reported in studies in the United States, where printed street maps typically follow this convention. This degree of consistency of depiction is important in the present context in that it allowed us to analyze the sketch maps using space-syntax axial-mapping techniques without a need to make any special assumptions about how to draw axial lines. There were, however, a number of key methodological challenges, and the next section describes the approach we took to resolve these.

\section{ATTRIBUTION OF THE SYNTACTIC VALUES OF A}

SKETCH MAP TO AN AXIAL MAP OF THE REAL WORLD

In this study, two techniques were used to elicit cognitive information from sketch maps. First, a conventional analysis was performed by disaggregating depicted elements. The number of times each element was drawn was counted. Second, a method has been developed for the analysis of global configurational aspects in sketch maps. Space-syntax analysis was applied directly to the sketch maps so that the syntactic characteristics of the whole map, considered as a configuration of spaces and features, could be quantified.

Our interest here was to analyze the errors in depiction and in omission as though these were realistic features of the respondent's cognition of the environment. Thus, if streets were consistently drawn wider than in reality, it would lead to fewer axial lines needed to go around a curve and similarly so if curves were consistently straightened. From the 76 samples, 3 sketch maps 


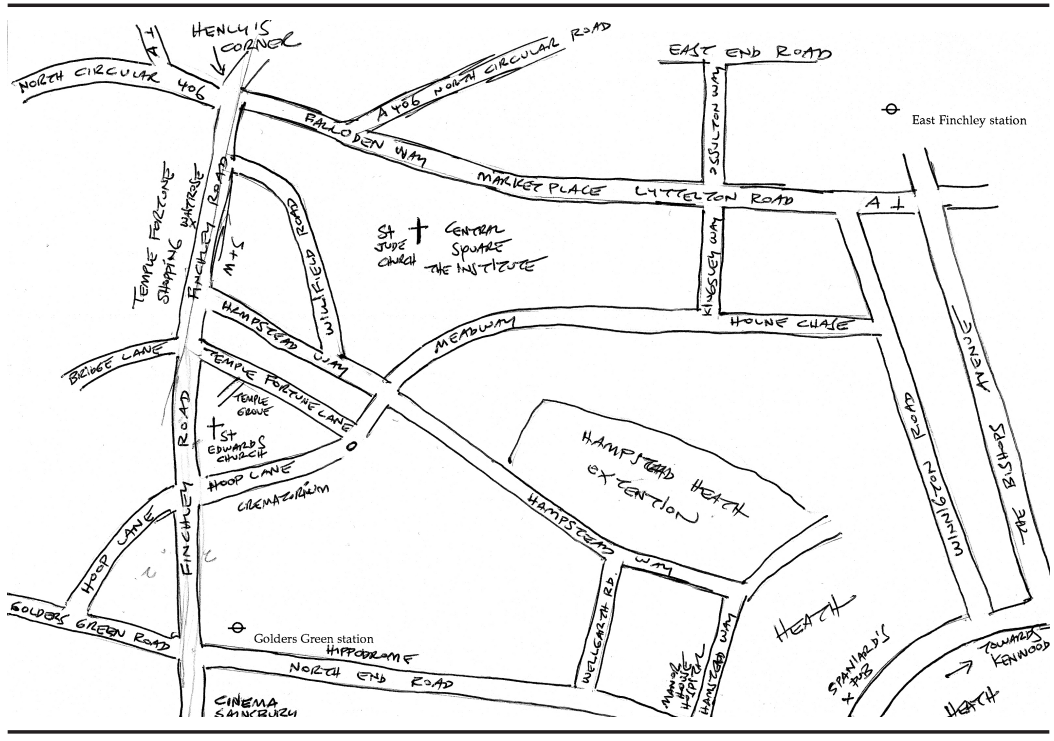

Figure 4a: Sample of a Well-Drawn Sketch Map

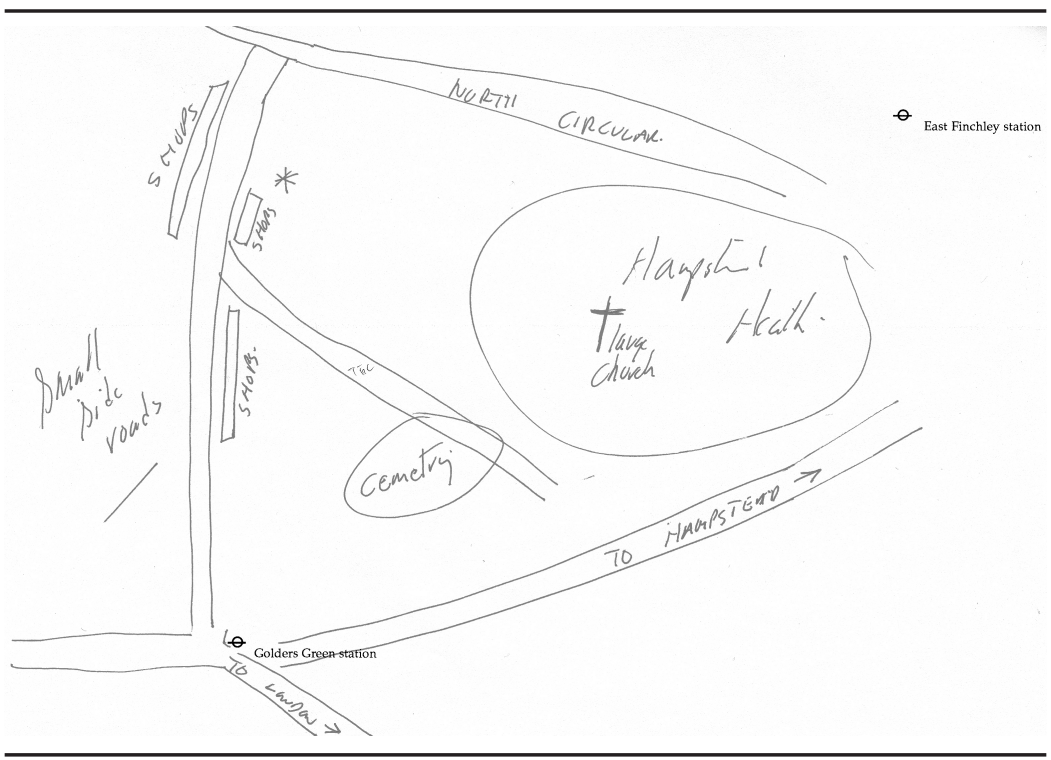

Figure ab: Sample of a Poorly Drawn Sketch Map 

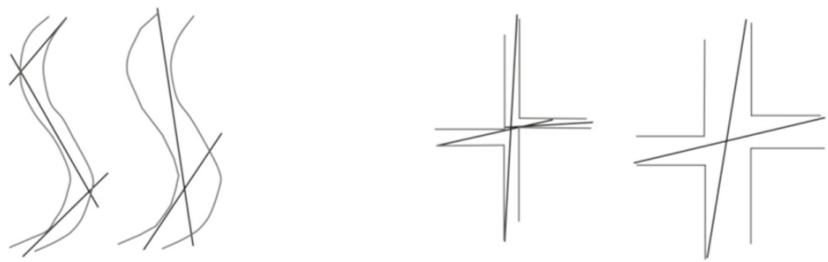

(a) Widening streets

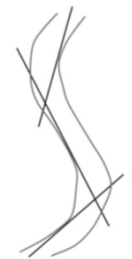

(b) Straightening of curved streets

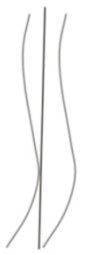

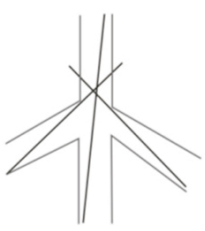

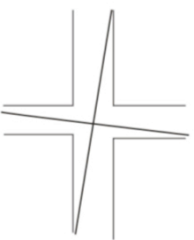

(c) Squaring of non-perpendicular interserions

Figure 5: Distortions of Reality in Sketch Maps

were excluded from the analysis because 2 represented streets as single lines and 1 had discontinuous parts, which are undigitizable for space-syntax analysis as a single system. Seventy-three sketch maps were digitized, and axial analysis was carried out to measure their syntactic characteristics.

The value of integration and of local integration was calculated for each space represented in the 73 respondents' sketch maps. The main aim of this analysis was to allow a statistical comparison between the configurations represented in the sketch maps of local residents and the configuration of the real-world map. This was achieved by transcribing configurational values of spaces featured in the sketch maps into a single statistical database. Because the method of analysis of sketch maps raises a number of methodological issues, the following sections describe this in more detail.

\section{SYNTACTIC ANALYSIS OF SKETCH MAPS}

Sketch maps generally contain errors and distortions and thus do not represent the real world exactly. Most commonly, distortions are in generic aspects of the maps such as widening a street, straightening of a curved street, 
orthogonalizing of nonperpendicular intersections (as shown in items a, b, and $\mathrm{c}$ in Figure 5), and omitting less important spaces. Left and right figures in items a, b, and c in Figure 5 represent those in the real world and in sketch maps, respectively. All these distortions tend to result in fewer axial lines in sketch maps than in the axial map of the real environment. Thus, sketch maps generally have fewer line segments to represent a street and are more integrated overall than the axial map of the real area. In practice, this factor simplifies the matter of attribution of sketch-map data to a statistical database to allow analysis of the degree of similarity and the divergence between the sketch map and the real world configuration.

Following the syntactic analyses of sketch maps, each line has its own set of syntactic values. The next task is to translate syntactic values measured on the sketch maps to the axial map of the real world. This is necessary to investigate the statistical relationship between syntactic properties of sketch maps and those of the real area. The procedure involves defining which axial lines in sketch maps correspond to which lines of the real map. This translation is done by examining sketch-map features such as intersections, the street layout, landmarks and, where present, street names. Because the sketch maps tend to be simplifications of the real map, one tends only to have to make inferences about which real street a sketched street is supposed to represent, and in the sample studied here, we did not encounter any fictitious streets on the sketch maps.

Although the procedure shows a correspondence between the axial maps of sketch maps and that of the real map, some confusion may arise, especially when numbers of axial lines representing a street are different (e.g., where the sketch map straightens a street that is curved in the real map). By investigating respondents' sketch maps, it was found that there are generally two ways to depict a street. One is to draw a whole street, and the other is to draw only a part of a street. When a whole street is drawn, the number of axial lines can differ compared to those of the real map. The number of axial lines can, in principle, be less than, the same as, or greater than reality. For example, Finchley Road consists of three axial lines in reality. However, it is generally less than three lines in the sketch maps. The general rules we applied in this study are explained in Figure 6 with possible examples.

The method described above was applied to all 73 sketch maps, and thus, each axial line in reality had a number of syntactic values based on its appearance in sketch maps. These were then averaged. For example, Finchley Road is depicted 67 times in all 73 sketch maps. Accordingly, the syntactic value of Finchley Road is produced by taking the mean of 67 syntactic values in sketch maps. If an axial line did not appear in any sketch map, it was excluded from the statistical analysis. 
Finchley Road 2

TABLE 2

Correlation Coefficients Between the Frequency and Syntactic Values of the Environment

TABLE 3

The Association Between Spatial Configuration in the Environment and in Sketch Maps

\begin{tabular}{lll}
\hline Item & $\mathrm{r}$ & $\mathrm{r}^{2}$ \\
\hline $\begin{array}{c}\text { global integration of sketch maps; } \\
\quad \text { global integration of real map }\end{array}$ & .486 & .236 \\
$\begin{array}{c}\text { global integration of sketch maps; } \\
\quad \text { local integration of real map }\end{array}$ & .648 & .42 \\
$\begin{array}{c}\text { local integration of sketch maps; } \\
\text { global integration of real map }\end{array}$ & .7 & .49 \\
$\begin{array}{l}\text { local integration of sketch maps; } \\
\text { local integration of real map }\end{array}$ & .728 & .53 \\
\hline
\end{tabular}

\section{SPATIAL CONFIGURATION IN THE REAL WORLD AND SPATIAL COGNITION IN SKETCH MAPS}

A composite image of the suburb was drawn according to the number of times each feature was depicted on the sketch maps. Table 1 shows the frequency of paths by segments. Finchley Road 2 (see Figure 3 ) is the most often mentioned, at $79.5 \%$ (58 out of 73 sketch maps).

Table 2 shows the correlations between the frequency with which features were represented in sketch maps and the syntactic variables describing the 


\begin{tabular}{|c|c|c|}
\hline Axial lines in reality & $\begin{array}{c}\text { Axial lines } \\
\text { in sketch maps }\end{array}$ & Interpretation \\
\hline b & & $\begin{array}{l}\text { A street is drawn as one axial line } \\
\text { although it is in three lines in reality. } \\
\text { In this case, all segments ( } a, b, c) \\
\text { get same syntactic values } \\
\text { processed by sketch map. }\end{array}$ \\
\hline$D$ & & $\begin{array}{l}\text { A street is drawn in two lines. } \\
\text { By examining a sketch map line-1 } \\
\text { represents line-a, b and line-2 } \\
\text { depicts line-c. Thus (a) and (b) } \\
\text { have same syntactic values of line- } \\
\text { 1, and line-c gets a value of line-2. }\end{array}$ \\
\hline$b$ & & $\begin{array}{l}\text { Only a part of a street is drawn in } \\
\text { sketch map. } \\
\text { Line-1 represents line-a, and } \\
\text { whether it covers (b) or up to (c) is } \\
\text { decided by examining a sketch } \\
\text { map. }\end{array}$ \\
\hline b & & $\begin{array}{l}\text { A street is drawn in more segments } \\
\text { in sketch maps than those of reality. } \\
\text { This case was never encountered in } \\
\text { this study. } \\
\text { Line-c is drawn in two lines ( } 3 \text { and } \\
\text { 4) in sketch maps, thus syntactic } \\
\text { values of line-c takes average value } \\
\text { of the two lines. }\end{array}$ \\
\hline
\end{tabular}

Figure 6: Translation of Syntactic Properties From Sketch Map to Axial Map

location of those features derived from an axial analysis of the real map. Table 2 shows that the frequency of depiction is generally correlated with the syntactic characteristics. Local integration shows the best correlation with frequency at $r=0.708(p<.0001)$. Figure 7 shows this correlation as a scattergram. The scatter is messy at the lower end of the distribution, but a highly significant positive relationship can be clearly seen between the two variables. Regression analysis confirms this with an $r^{2}$ value of $0.501(p<.0001)$ showing that if a space is locally integrated, it tends to be depicted more often 


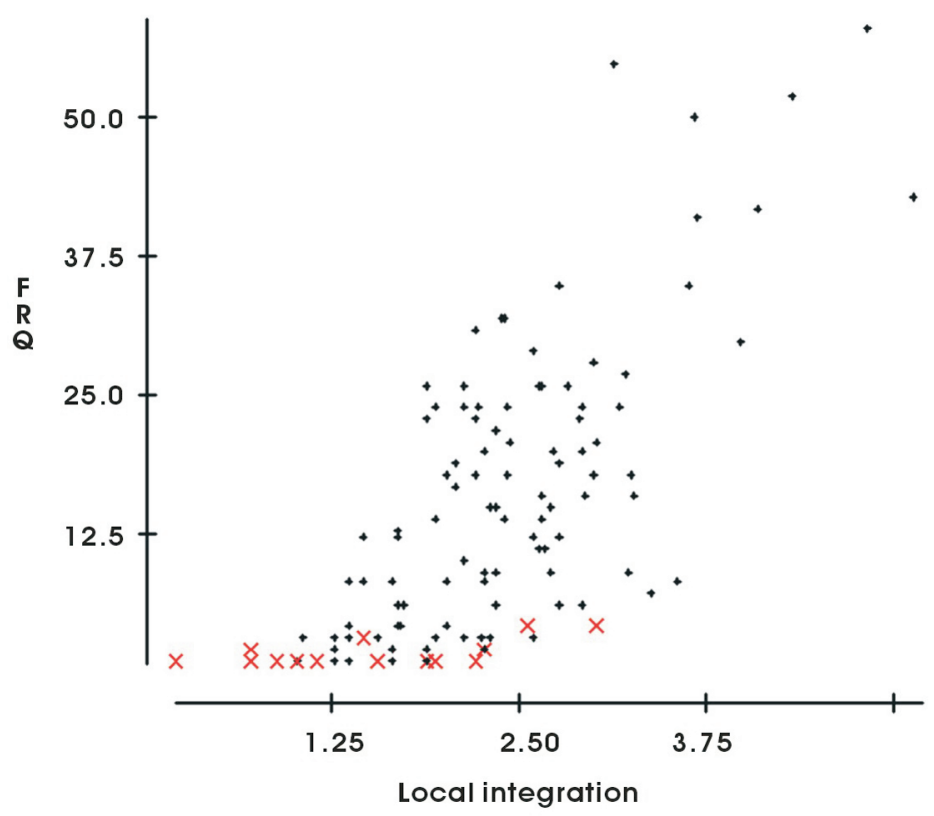

Figure 7: Scattergram Between Frequency and Local Integration

in sketch maps. Most spaces that appear with low frequency are cul-de-sacs and footpaths, which are represented as $x$ in the scatter. Features of this sort are less spatially integrated and less frequently cited. This result suggests that spatial configuration may play a direct role in the acquisition of knowledge of the environment.

To investigate this further, our analysis looked not only at the frequency with which features are represented in sketch maps but also at the configurational properties of the maps. The syntactic properties of the sketch maps were quantified using axial-analysis methods in the same way that these had been used for maps of the real world. The mean global integration and the local integration of each space depicted were calculated for all 73 sketch maps. Next, the syntactic variables for each space represented in the sketch maps were attributed to the representation of that space in the axial map of the real world. Figure 8, for example, displays the mean local integration of spaces calculated on the basis of all 73 sketch maps displayed on the real axial map of the suburb using a scale from black for the highest integration to light 


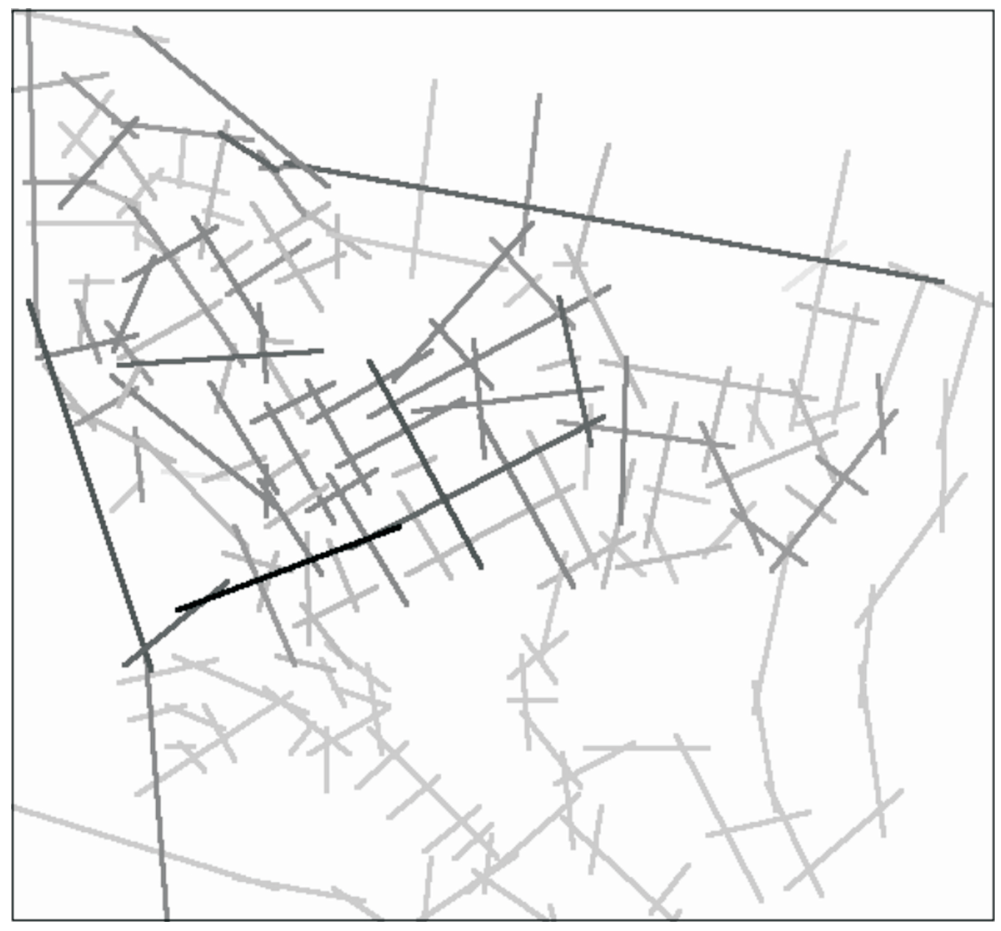

Figure 8: Mean Local Integration of the Suburb in Sketch Maps

gray for the lowest. The pattern of integration is similar to the local integration for the real map of the suburb, as shown in Figure 3.

Table 3 shows correlation and regression analyses between syntactic variables from the real world and from the sketch-map analysis, respectively. Global integration in the sketch map and local integration in reality are well correlated at $r=0.648(p<.0001)$ (see Figure 9). The regression analysis gives an $r^{2} 0.42(p<.0001)$. Similarly, the coefficient between local integration in the sketch map and local integration in reality is also high at $r=0.7(p$ $<.0001)$ (see Figure 10). The regression analysis gives an $r^{2}$ of $0.49(p<$ .0001). A close examination of the scatters in Figures 9 and 10 reveals two outlier groups, one in the upper right part, represented as an $x$, and another in the lower left, marked as $o$. The first outlier group consists of spaces on the boundary of the suburb, and the second group consists of the cul-de-sacs throughout the area. The boundaries have a high local-integration value, but 


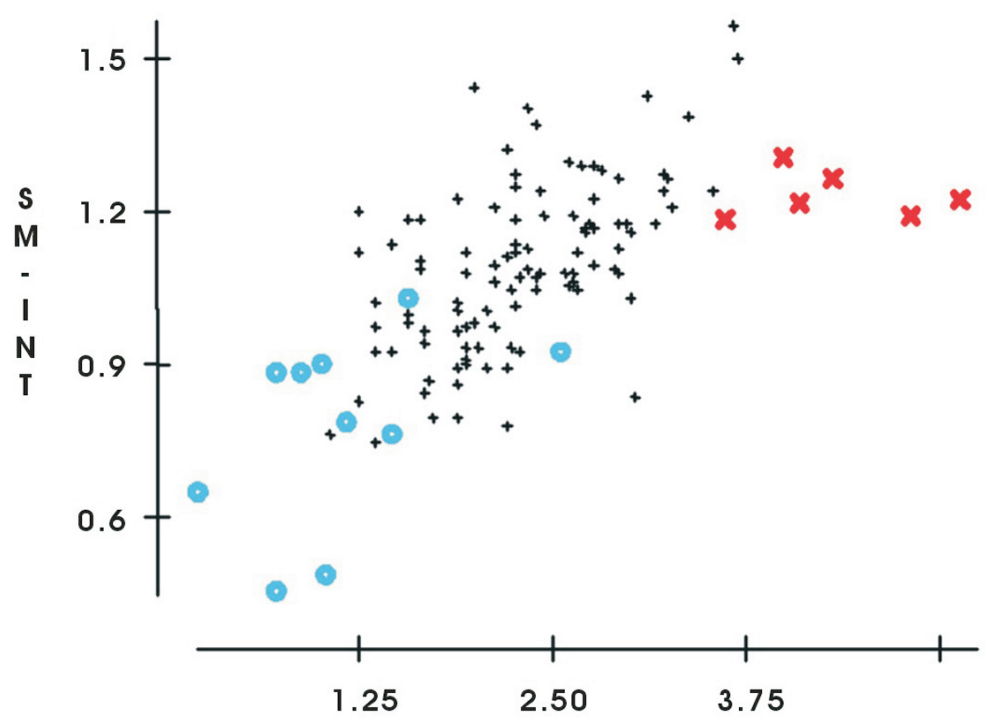

Local integration

Figure 9: Scattergram Between (Vertical) Global Integration of Sketch Maps and (Horizontal) Local Integration of Reality

these spaces were not so frequently represented on the sketch maps. Conversely, cul-de-sacs are characterized not only by low local integration in reality but also by their low frequency of appearance on the sketches.

It can be concluded that there is a clear pattern of association between the syntactic properties of the real environment and those of sketch maps, suggesting that the measure of local integration is a better predictor of cognitive representations of spatial configuration than is global integration. This suggests that a positive relationship exists between spatial configuration in the real environment and its cognitive representation. The local integration of the real map shows a slightly better correlation with local integration of the sketch maps than with global integration. Taken alongside the finding that local integration is most closely related to the frequency of features drawn on the sketch maps, this suggests that local integration is strongly related not only with simple information referring to the appearance of features in sketch maps but also with the more complex information referring to relational knowledge of spatial configuration. 


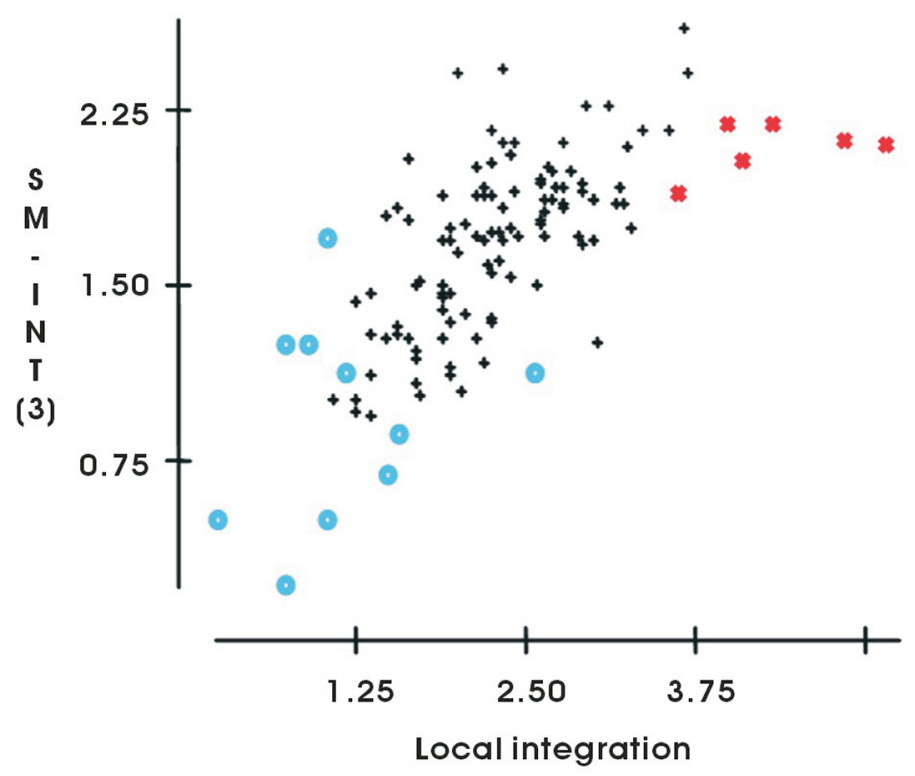

Figure 10: Scattergram Between (Vertical) Global Integration of Sketch Maps and (Horizontal) Local Integration of Reality

\section{DISCUSSION AND CONCLUSIONS}

This article has uncovered some underlying regularities in the relationship between spatial configuration in the real world and aspects of spatial cognition derived from sketch-map data. Several points can be concluded from the association revealed by the analysis. Firstly, a positive relationship is found between the spatial configuration in the real world and its representation in spatial cognition (so far as that can be elicited through sketch mapping). The frequency with which configurational elements are identified on the sketch maps is highly correlated with all the syntactic measures. The syntactic properties of spatial configuration represented on the sketch maps are also associated with syntactic variables in the real world. Secondly, local integration, the syntactic property that is derived from the relational properties of spaces in the immediate environment, shows the best relationship with the sketch-map variables, such as the frequency with which features are represented and the local integration of the sketch map treated as a spatial system in its own right. 
All this evidence suggests that in spite of the well-documented errors and inconsistencies found in sketch maps, the image of spatial configuration maintained in cognitive maps is highly associated with that of the real, physical, built environment. In some sense, this is no surprise-configurational and relational properties of spatial patterns are perhaps more important for the purposes of navigation than is precise local information.

These findings have implications for the investigation of the relationship between man and the built environment, both methodologically and theoretically. From a methodological perspective, previous approaches to the analysis of sketch-map data, such as those developed by Lynch (1960), which disaggregate depicted features into five categories (paths, edges, districts, nodes, and landmarks) have focused on the analysis of cognitive representation. However, an obvious problem with respect to his analysis is that because it is based on a disaggregation of represented spatial elements, it cannot account for the configurational or for the relational properties of spatial environments in spite of the admitted importance of continuity and of the interrelationship of elements in evoking a strong image. In this context, this study developed space-syntax analysis of sketch maps, and it allowed us to investigate the effects of spatial configuration on spatial cognition by analyzing relational aspects of spatial configuration in sketch maps.

The findings presented here also seem to have some theoretical interest. First, a strong correlation is found between sketch maps and purely configurational measures of spatial patterns. No account has been taken of the location of attractors or of landmarks in constructing this relationship. The positive association lends strong support to the conjecture that spatial configuration is at the root of the way we cognize built environments. The cognition of spatial configuration appears to result naturally from the way the spatial configuration of the physical world is ordered. Second, the transmission of configurational knowledge from the built environment can be facilitated and/ or impeded by spatial configuration. As Golledge and Stimson (1997) pointed out, spatial knowledge consists of two complementary sets in the process of spatial learning in everyday experience: (a) the knowledge of global structure at a cognitive level, resulting from long-term exposure to the built environment, and (b) the intuitive knowledge of a more immediate environment at a perceptual level. The frequency in sketch maps seems to refer to a simple and first phase of spatial knowledge, which is often referred as landmark or cue knowledge (Shemyakin, 1962). On the other hand, the spatial syntax of sketch maps, which represent and quantify relational or configurational knowledge to some degree, may refer to more complex knowledge of spatial layout. Golledge and Stimson (1997) described this kind of knowledge as the knowledge of hierarchical networks, chunking, and an understanding of 
the notion of configuration in a multidimensional sense. As has been found in this research, these two aspects of spatial knowledge are positively affected by the spatial configuration of the real environment.

In conclusion, this research has shown how the syntactic description of spatial configuration and the theoretical positions of sketch maps can be combined in an integrated approach in investigating the interaction between spatial configuration and spatial cognition. This incorporation of spatial syntax of spatial configuration implies a specific conception in understanding human spatial experience.

\section{NOTES}

1. There have been a number of studies of aspects of cognition within the space-syntax community. See, in particular, Peponis, Zimring, and Choi (1990), Choi (1991), and Conroy (2001). However, in the main, these studies have been based on experimental- and on observation-based methods rather than on the sketch-mapping techniques that form the basis for the work described in this article (see Penn, 2001, for a review).

2. For a detailed description of the process see Hillier and Hanson (1984, pp. 91-92).

3. See Hillier and Hanson (1984, pp. 109-113) for a full treatment of this relativization.

\section{REFERENCES}

Appleyard, D. (1970). Styles and methods of structuring a city. Environment \& Behavior, 2, 100124.

Choi, Y. K. (1991). The spatial structure of exploration and encounter in museum layouts. Unpublished doctoral dissertation, Georgia Institute of Technology, Atlanta.

Conroy, R. A. (2001). Spatial navigation in immersive virtual environments. Unpublished doctoral dissertation, University of London, London.

Downs, M., \& Stea, D. (1973). Cognitive maps and spatial behavior: Process and products. In R. M. Downs \& D. Stea (Eds.), Image and environment (pp. 8-26). London: Arnold.

Evans, G. W., \& Pezdek, K. (1980). Cognitive mapping: Knowledge of real-world distance and location information. Journal of Environmental Psychology, 6, 13-24.

Garling, T., Lindberg, C. M., Book, A. (1986). Reference systems in cognitive maps. Journal of Environmental Psychology, 6, 1-18.

Golledge, R. G., \& Stimson, R. (1997). Spatial behavior: A geographic perspective. New York: Guilford.

Hart, R. A., \& Moore, G. T. (1973). The development of spatial cognition: A review. In R. M. Downs \& D. Stea (Eds.), Image and environment (pp. 246-288). London: Arnold.

Hillier, B. (1996). Space is the machine. UK: Cambridge University Press.

Hillier, B., \& Hanson, J. (1984). The social logic of space. UK: Cambridge University Press.

Lynch, K. (1960). The image of the city. Cambridge, MA: MIT Press. 
O’Keefe, J., \& Nadel, L. (1978). The hippocampus as a cognitive map. UK: Oxford University Press.

O’Neill, M. J. (1991). Effects of signage and floor plan configuration on wayfinding accuracy. Environment \& Behavior, 23, 553-574.

Penn, A. (2001, May). Space syntax and spatial cognition: Or why the axial line? Paper presented at the Third International Space Syntax Symposium, Georgia Institute of Technology, Atlanta, GA.

Peponis, J., Zimring, C., \& Choi, Y. K. (1990). Finding the building in wayfinding. Environment \& Behavior, 22, 555-590.

Pipkin, J. S. (1981). Cognitive behavioural geography and repetitive. In K. R. Cox \& R. G. Golledge (Eds.), Behavioral problems in geography revisited (pp. 145-181). New York: Methuen.

Sadalla, E. K. \& Magel, S. G. (1980). The perception of traversed distance. Environment and Behavior, 12, 65-79.

Sadalla, E. K. \& Montello, D. (1989). Remembering changes in direction. Environment and Behavior, 21, 346-363.

Sadalla, E. K., \& Staplin, L. G. (1980). The perception of traversed distance: Intersections. Environment and Behavior, 12, 167-182.

Shemyakin, F. N. (1962). General problems of orientation in space and space representations. In B. G. Anan'yev (Ed.), Psychological science in the USSR (NTIS Report No. TT62-11083; Vol. 1, pp. 184-225). Washington, DC: Office of Technical Services.

Weisman, G. (1981). Evaluating architectural legibility: Wayfinding in the built environment. Environment \& Behavior, 13, 189-204.

Zimring, C. M. (1981). Stress and the designed environment. Journal of Social Science, 37, 145171. 